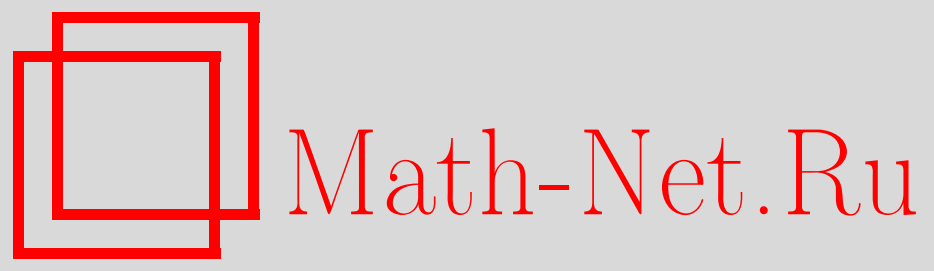

Ю. А. Кашлев, Две стадии движения ангармонических осцилляторов, моделирующих быстрые частицы в кристаллах, ТМФ, 2011, том 167, номер 1, 123-135

DOI: https://doi.org/10.4213/tmf6629

Использование Общероссийского математического портала Math-Net.Ru подразумевает, что вы прочитали и согласны с пользовательским соглашением http://www.mathnet.ru/rus/agreement

Параметры загрузки:

IP : 52.6 .47 .48

26 апреля 2023 г., 15:13:43

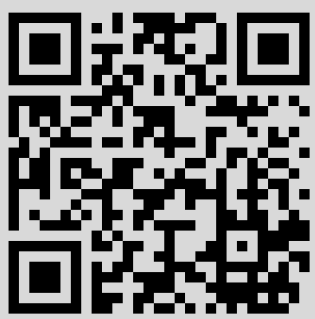




\title{
(C) 2011 г. \\ ДВЕ СТАДИИ ДВИЖЕНИЯ АНГАРМОНИЧЕСКИХ ОСЦИЛЛЯТОРОВ, МОДЕЛИРУЮЩИХ БЫСТРЫЕ ЧАСТИЦЫ В КРИСТАЛЛАХ
}

\begin{abstract}
Рассмотрена эволюция пространственного распределения быстрых атомных частиц по мере прохождения их через кристалл. На малых глубинах проникновения, когда частицы движутся без значительной потери когерентности, происходит сглаживание совместной плотности распределения вероятности за счет градиента негармонического потенциала плоскостного канала. Показано, что в результате сглаживания устанавливается квазиравновесное (квазистационарное) состояние подсистемы быстрых частиц. Дальнейшая эволюция распределения частиц обусловлена неупругим рассеянием и описывается с помощью кинетического уравнения. На этой стадии негармоничность колебаний частиц между стенками канала приводит к перенормировке полного сечения фононного рассеяния частиц, причем перенормировка определяется ангармоническим взаимодействием четвертого порядка.
\end{abstract}

Ключевые слова: быстрая атомная частица, ангармонический осциллятор, кристалл, фонон, канал, каналированная частица, уравнение Лиувилля, кинетическое уравнение, неупругое рассеяние, ангармонизм четвертого порядка.

\section{1. ВВЕДЕНИЕ}

Согласно классической теории Линдхарда [1] при углах влета в канал, меньших критического угла каналирования $\psi_{\mathrm{c}}$, пучок быстрых атомных частиц разделяется на две части: каналированную и хаотическую. Каналированные частицы (КЧ) движутся в центре плоскостного канала, совершая при этом периодические отражения от кристаллографических плоскостей, образующих стенки канала. В отличие от КЧ хаотические частицы движутся по прямым траекториям, пересекающим плоскости каналов под произвольными углами.

При рассмотрении эффекта каналирования различают две стадии процесса: стадию сглаживания распределения частиц, на которой формируется режим каналирования высокоэнергетических частиц, и стадию колебательной релаксации ангармонических осцилляторов, моделирующих КЧ.

${ }^{*}$ Институт металлургии и материаловедения им. А. А. Байкова РАН, Москва, Россия 
В настоящей работе мы исследуем обе указанные стадии с учетом вклада ангармонизма колебаний частиц между стенками канала.

\section{2. СГЛАЖИВАНИЕ ПЛОТНОСТИ РАСПРЕДЕЛЕНИЯ ЧАСТИЦ НА МАЛЫХ ГЛУБИНАХ ПРОНИКНОВЕНИЯ}

Будем изучать временну́ю эволюцию пространственного распределения частиц, падающих на кристалл под малым углом $\psi_{0}\left(\right.$ естественно, $\left.\psi_{0}<\psi_{\mathrm{c}}\right)$ к кристаллографической плоскости. В рамках классической теории можно считать, что начальная угловая расходимость пучка много меньше критического угла каналирования, $\delta \psi_{0} \ll \psi_{\text {c. }}$ Взаимодействие быстрой частицы с энергией $E_{0}$ с кристаллом будем описывать как когерентное воздействие большого числа атомов кристаллографической плоскости, другими словами, с помощью непрерывного негармонического потенциала плоскости $U_{\mathrm{pl}}(x)[1]$. Отметим также, что поток быстрых частиц падает на кристалл в $z$-направлении.

Будем изучать эволюцию на малых глубинах, используя уравнение Лиувилля. На этих глубинах неупругое рассеяние неэффективно, и фазовое перемешивание обусловлено градиентом негармонического потенциала:

$$
C(x)=\frac{1}{2 E_{0}} \frac{\partial}{\partial x} U_{\mathrm{pl}}(x) .
$$

Если $\vartheta$ - угол между направлением скорости частицы и кристаллографической плоскостью $(y, z)$, то уравнение Лиувилля для распределения частиц по поперечной координате $x$ и поперечной скорости $v_{x}$, точнее, по углу $\vartheta=v_{x} / v_{z}$ имеет вид

$$
\frac{\partial}{\partial z} \sigma(\tilde{x}, \vartheta, z)+\vartheta \frac{\partial}{\partial \tilde{x}} \sigma(\tilde{x}, \vartheta, z)-C(\tilde{x}) \frac{\partial}{\partial \vartheta} \sigma(\tilde{x}, \vartheta, z)=0
$$

где $\tilde{x}$ отсчитывается от середины канала. Наша задача сводится к решению этого уравнения при начальном и граничном условиях

$$
\left.\sigma(\tilde{x}, \vartheta, z)\right|_{\tilde{x}=0, z=0}=\sigma_{0}(\vartheta),\left.\quad \sigma(\tilde{x}, \vartheta, z)\right|_{\tilde{x}=0}=\left.\sigma(\tilde{x}, \vartheta, z)\right|_{\tilde{x}=2 l} .
$$

Периодическое граничное условие обусловлено тем, что потенциал системы параллельных плоскостей $U_{\mathrm{pl}}(x)$ является периодической функцией в $x$-направлении с периодом, равным расстоянию $2 l$ между плоскостями.

Мы решали уравнение (1) численно, причем в качестве потенциала $U_{\mathrm{pl}}(x)$ был взят непрерывный плоскостной потенциал, соответствующий потенциалу атом-атомного взаимодействия в форме Мольер [2]. При решении уравнения (1) нами использовался подход из работы [3], согласно которому расчет велся по двухслойной неявной схеме бегущего счета, устойчивой при указанном выше начальном условии. Что касается распределения $\sigma_{0}(\vartheta)$, вошедшего в начальное условие, то оно было взято в гауссовой форме:

$$
\sigma_{0}(\vartheta)=\frac{1}{\sqrt{2 \pi\left(\delta \psi_{0}\right)^{2}}} \exp \left\{-\frac{\left(\vartheta-\vartheta_{0}\right)^{2}}{2\left(\delta \psi_{0}\right)^{2}}\right\} .
$$

Рассмотрим график плотности распределения частиц

$$
\rho(x, z)=\int d \vartheta \sigma(x, \vartheta, z)
$$




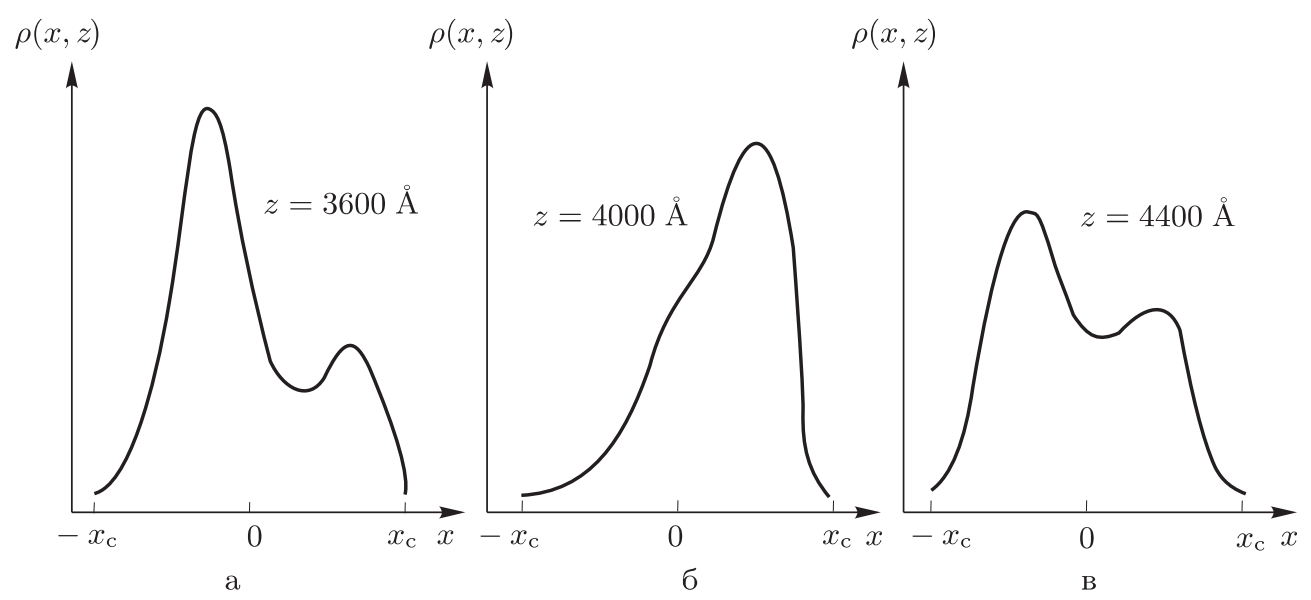

Рис. 1. Плотность распределения частиц на разных глубинах проникновения протонов.

при разных глубинах проникновения протонов с энергией $E_{0}=0.5 \mathrm{M \ni В} \mathrm{в} \mathrm{плоскост-}$ ном канале (100) монокристалла кремния при температуре $300 \mathrm{~K}$ (все остальные графики даны для тех же частиц и тех же условий движения). Следует иметь в виду, что частицы, вошедшие под углом $\psi_{0}<\psi_{\mathrm{c}}$, не приближаются к стенке канала ближе, чем на радиус экранирования Томаса-Ферми $a_{\text {тF }}$ [1]. Поэтому можно ввести критическое отклонение быстрой частицы от центра канала $x_{\mathrm{c}}=l-a_{\mathrm{TF}}$.

Результаты численного интегрирования уравнений (1) и (2) при $\vartheta_{0}=\psi_{\mathrm{c}} / 2$ представлены на рис. 1, где показана плотность распределения (в свободных единицах) на трех глубинах: $z=3600 \AA$ (а), $z=4000 \AA$ (б), $z=4400 \AA$ (в).

Согласно рис. 1 распределение на малых глубинах осциллирует между стенками канала, и высота максимумов функции $\rho(x, z)$ уменьшается с ростом глубины. Указанное сглаживание осцилляций обусловлено негармоничностью потенциала, вошедшего в величину $C(x)$.

Если потенциал канала аппроксимировать гармонической функцией, то получаемая при этом плотность будет периодической функцией с длиной волны $\tilde{\Lambda}$. В этом случае естественно определить квазиравновесное (квазистационарное) значение плотности $\overline{\rho(x)}$ как решение уравнения (1), усредненное по длине волны,

$$
\overline{\rho(\tilde{x})}=\lim _{\tilde{\Lambda} \rightarrow \infty} \frac{1}{\tilde{\Lambda}} \int_{0}^{\tilde{\Lambda}} d z \int d \vartheta \sigma(\tilde{x}, \vartheta, z) .
$$

В гармоническом приближении траекторией частицы в поперечном пространстве $\left(x, p_{x}\right)$ является эллипс. Даже пренебрежимо малое воздействие негармоничности приводит к вращению этого эллипса в поперечном пространстве, в результате чего траектории частицы равномерно заполняют всю доступную область. Полученная таким образом средняя плотность в $x$-пространстве, как показал анализ, равна

$$
\overline{\rho_{\mathrm{har}}(\tilde{x})}=\frac{1}{\pi} \theta\left(d_{\mathrm{c}}-\tilde{x}\right) \ln \left\{\frac{\left|x_{\mathrm{c}}^{2}-\chi\right|^{1 / 2}+\left|x_{\mathrm{c}}^{2}-\tilde{x}^{2}\right|^{1 / 2}}{\left|\tilde{x}^{2}-\chi\right|^{1 / 2}}\right\},
$$




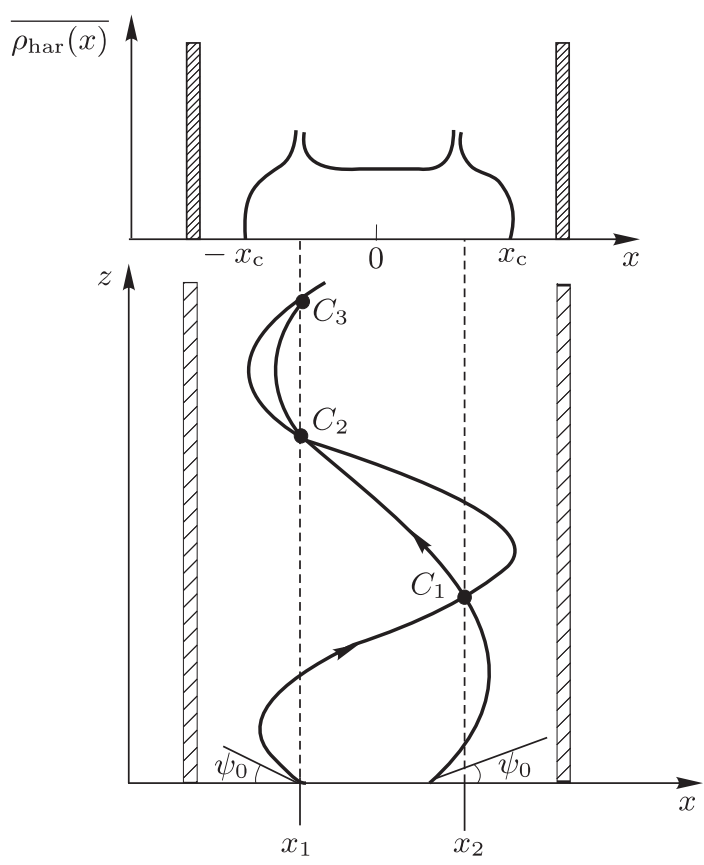

Рис. 2. Квазиравновесная (квазистационарная) плотность в гармоническом приближении (вверху) и траектории частиц (внизу).

причем данная функция имеет сингулярности в точках $\tilde{x}_{1}$ и $\tilde{x}_{2}$, координаты которых удовлетворяют уравнению $U_{\mathrm{pl}}\left(\tilde{x}_{m}\right)=E_{0} \psi_{0}^{2}, m=1,2$, и в случае плоскостного канала $\tilde{x}_{1}=-\tilde{x}_{2}$. Кроме того, использовано обозначение $\chi=\tilde{x}_{1}^{2}=\tilde{x}_{2}^{2}, \theta(x)$ - тета-функция.

Нетрудно дать объяснение указанных особенностей функции $\rho_{\mathrm{har}}(\tilde{x})$ в рамках классической теории, рассматривая траектории частиц (см. рис. 2). Действительно, частицы, вошедшие в канал под одним и тем же углом к стенке, могут иметь разные амплитуды колебаний, если они влетают с разными прицельными расстояниями. Траектории, имеющие разные амплитуды, пересекаются, что продемонстрировано на рис. 2. Образующиеся в результате точки пересечения $C_{1}, C_{2}, C_{3}$ определяют те значения $\tilde{x}_{1}$ и $\tilde{x}_{2}$ на оси $x$, в которых наблюдаются сингулярности. Разумеется, пересекаются не отдельные траектории, как условно показано на рис. 2, а множество траекторий, поэтому в действительности пики плотности заменяются на максимумы конечной высоты. Отметим, что заметные пики плотности вблизи стенок канала (flux peaking effect) возникают и в квантовой теории каналирования [4], где их связывают с наличием частиц на надбарьерных уровнях.

Остановимся более подробно на частном случае, а именно на затухании осцилляций распределения в центре канала. Для этого прежде всего запишем градиент плоскостного потенциала в явном виде, предполагая, что быстрые частицы движутся в "замороженной" решетке с фиксированными отклонениями атомов.

Если непрерывный потенциал плоскости вычисляется на основе атом-атомного потенциала Мольер, то его градиент имеет вид

$$
\frac{d}{d x} U_{\mathrm{pl}}(x)=\frac{Z_{1} Z_{2} e^{2}}{d}\left\{0.1 R_{1}(x)+0.55 R_{2}(x)+0.35 R_{3}(x)\right\} .
$$


Здесь

$$
\begin{gathered}
R_{i}(x)=-\frac{\beta_{i}}{a_{\mathrm{TF}}} \int_{\xi_{i}(x)}^{\infty} d \rho \exp \left(\beta_{i} \tilde{a}-\beta_{i} \frac{x}{a_{\mathrm{TF}}} \sqrt{1+\rho^{2}}\right)+\frac{1}{x} \exp \left(-\frac{1}{4 \tilde{a}} \frac{x^{2}}{a_{\mathrm{TF}}^{2}}\right), \\
\xi_{i}(x)=\frac{1}{x} \beta_{i} \tilde{a} a_{\mathrm{TF}}-\frac{x}{4 \beta_{i} \tilde{a} a_{\mathrm{TF}}},
\end{gathered}
$$

$\tilde{a}=\sigma_{\theta}^{2}\left(2 a_{\mathrm{TF}}^{2}\right)^{-1}, \sigma_{\theta}$ - среднеквадратичное (тепловое) отклонение атома кристалла от узла; $Z_{1}$ и $Z_{2}$ - заряды быстрой частицы и атома кристалла; $d$ - ближайшее расстояние между атомами $z$-цепочки, вдоль которой движется быстрая частица; $\beta_{i}$ - численные множители, входящие в потенциал Мольер, $i=1,2,3$.

Введем безразмерный градиент в виде

$$
g(x)=\left(\frac{d U_{\mathrm{pl}}}{d x}\right)\left(\frac{a_{\mathrm{TF}}}{U_{0}}\right)
$$

где $U_{0}=Z_{1} Z_{2} e^{2} / d$, а переменная $x$ отсчитывается от стенки канала. Численный анализ выражения (5) показал, что $g(x)$ можно представить в виде суммы $g_{1}(x)+g_{2}(x)$, где $g_{1}(x)$ - линейная функция, соответствующая градиенту гармонического потенциала, а $g_{2}(x)$ - быстровозрастающая функция, которая в области ее максимального значения (вблизи стенки) может быть аппроксимирована восходящей ветвью кубической параболы. Последнее означает, что функция $g_{2}(x)$ соответствует градиенту ангармонического потенциала четвертого порядка. Введение в теорию ангармонизма четвертого порядка не нарушает симметрию задачи, но приводит к сужению энергетического расстояния между соседними уровнями потенциала канала по мере роста номера уровня. Эта неэквидистантность уровней учитывается в первую очередь при анализе колебательной релаксации в системе ангармонических осцилляторов [5].

С учетом обозначения $\mathcal{F}(x)=-\left(U_{0} / a_{\text {тF }}\right) g(x)$ для силы уравнение Лиувилля для плотности вероятности (1) запишем в следующем виде:

$$
\frac{\partial}{\partial z} \sigma(x, \vartheta, z)+\vartheta \frac{\partial}{\partial x} \sigma(x, \vartheta, z)+\frac{1}{2 E_{0}} \mathcal{F}(x) \sigma(x, \vartheta, z)=0 .
$$

Согласно (5) функция $\mathcal{F}(x)$ имеет достаточно сложный вид, что делает невозможным решение уравнения (6) аналитическими методами. Это решение может быть получено только методами численного интегрирования. Используя график $\sigma(x, \vartheta, z)$, нетрудно получить аппроксимирующую функцию для пространственной плотности частиц. Более того, мы можем ограничиться рассмотрением поведения плотности в центре канала, что значительно упрощает задачу.

Результат численного расчета в случае, когда протоны влетают в кристалл кремния вдоль стенки канала (100), $\psi_{0}=0$, представлен на рис. 3 (сплошная кривая). Как видно из рисунка, на всем интервале глубин, где формируется квазиравновесие в подсистеме частиц, функция $\rho(0, z)$ претерпевает осцилляции, затухающие с ростом глубины. Осциллирующее поведение функции $\rho(0, z)$ может быть аппроксимировано аналитической функцией

$$
\rho_{\mathrm{anh}}(0, z)=\overline{\rho_{\mathrm{har}}}\left\{1-\frac{3}{5} F_{1}(t) \cos \left(\omega_{\mathrm{ex}} t\right)-\frac{2}{5} F_{2}(t) \cos \left(2 \omega_{\mathrm{ex}} t\right)\right\},
$$




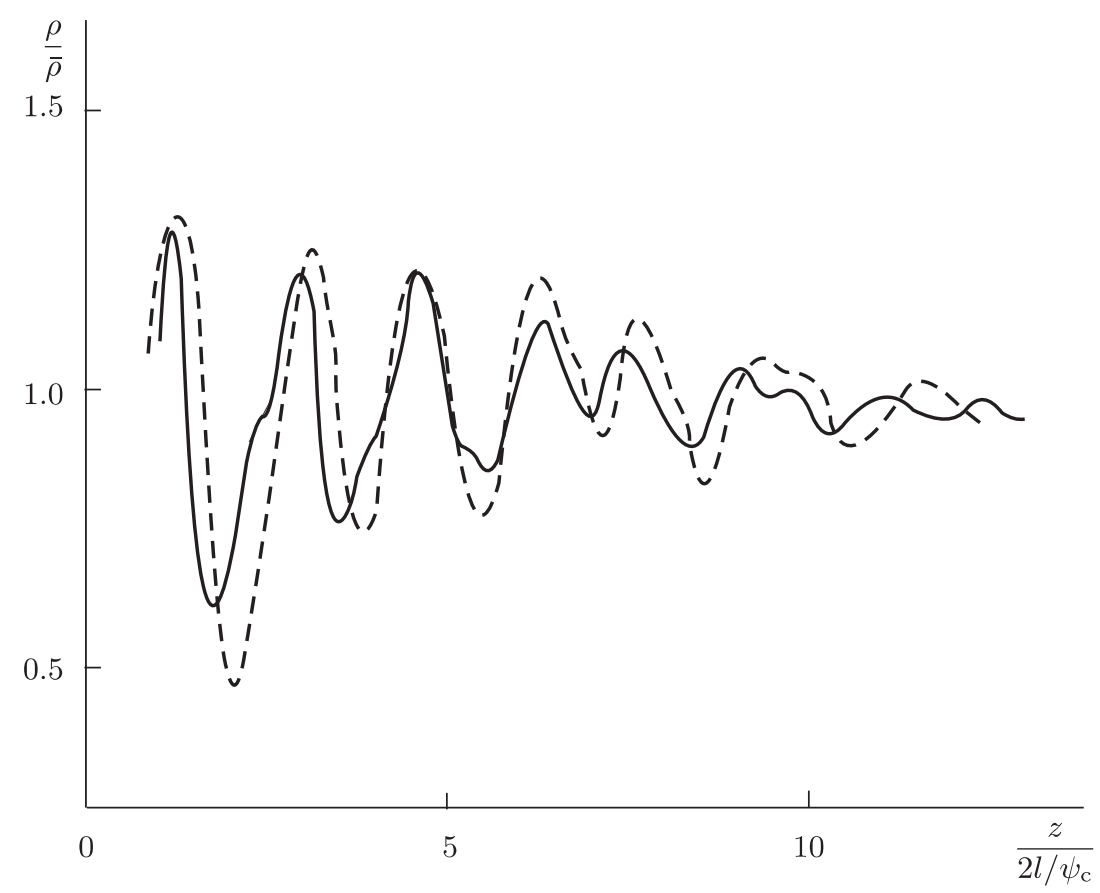

Рис. 3. Затухание осцилляций пространственного разделения частиц в центре канала с ростом глубины проникновения.

где $\omega_{\text {ex }}$ - частота обмена энергией между частицами пучка, в результате такого обмена происходит перераспределение поперечной энергии (в сопровождающей системе координат) по колебательным степеням свободы частиц [5]. Функции $F_{1}(t)$ и $F_{2}(t)$, определяющие затухание, удобно представить в гауссовой форме:

$$
F_{1}(t)=\exp \left\{-\gamma_{1} \frac{t^{2}}{\tau_{\mathrm{s}}^{2}}\right\}, \quad F_{2}(t)=\exp \left\{-\gamma_{2} \frac{t^{2}}{\tau_{\mathrm{s}}^{2}}\right\}
$$

где $\gamma_{1}$ и $\gamma_{2}$ - свободные параметры теории, $\tau_{\mathrm{s}}$ - время жизни колебательной моды между двумя последовательными "столкновениями" за счет ангармонического взаимодействия [6].

ЗАмЕчание 1 . Если ангармонизм не учитывается и $\gamma_{i}=0$, то $F_{1}(t)=F_{2}(t)=1$, плотность (7) становится периодической функцией без затухания. В этом случае усреднение $\rho_{\mathrm{anh}}(0, z)$ выполняется аналогично усреднению $(3)$, что дает плотность в гармоническом приближении.

ЗАмЕчАниЕ 2. Варьируя свободные параметры $\gamma_{1}$ и $\gamma_{2}$, можно достичь удовлетворительного согласия функции $\rho(0, z)(2)$, полученной путем численного решения уравнения (6) (см. сплошную кривую на рис. 3), с аппроксимирующей функцией $\rho_{\text {anh }}(0, z)(7)$ (см. штриховую кривую на том же рис.). Это дает возможность получить пространственную плотность частиц в аналитической форме. 
Оценим глубину $L$, на которой затухают осцилляции плотности, другими словами, глубину, на которой устанавливается квазиравновесное, квазистационарное распределение частиц. Для этого достаточно подставить в (7) значение плотности $\rho_{\mathrm{anh}}$, равное среднему $\overline{\rho_{\text {har }}}$ в гармоническом приближении, и решить полученное трансцендентное уравнение относительно $z=L$. Однако ту же оценку можно получить проще, а именно с использованием графика функции (7). Например, для протонов с энергией 0.5 МэВ в канале (100) кремния (штриховая кривая на рис. 3) получаем оценку

$$
L \simeq 13\left(\frac{2 l}{\psi_{\mathrm{c}}}\right) \simeq 5 \cdot 10^{3} \AA .
$$

Если эффект каналирования рассматривать в рамках квантовой статистической механики [4], то задача вычисления $L$ сводится к нахождению длины когерентности $L_{\text {coh }}$, на которой затухают недиагональные элементы матрицы плотности. Полученный таким путем результат $L_{\text {соһ }} \simeq 10^{4} \AA$ по порядку величины совпадает с классическим значением (8).

Этап сглаживания плотности (фактически, затухания осцилляций) завершается формированием распределения, зависящего от поперечной энергии

$$
\varepsilon_{\perp}=\frac{p_{x}^{2}}{2 m}+\frac{1}{2} m \Omega^{2} x^{2}
$$

Будем считать, что взаимодействие частиц с термостатом содержит малый параметр $\delta$. Выберем интервал времени $\Delta t$ так, что $\Delta t$ значительно больше периода $2 \pi / \Omega$ осцилляций частиц в канале. Кроме того, будем считать, что воздействие термостата за время $\Delta t$ пренебрежимо мало. Тогда в классической интерпретации совместная плотность вероятности $\sigma\left(x, p_{x}, t\right)$ трансформируется в $\sigma\left(\varepsilon_{\perp}, t\right)$ в том смысле, что [7]

$$
\frac{1}{\Delta t} \int_{t}^{t+\Delta t} d t^{\prime} \sigma\left(x, p_{x}, t^{\prime}\right) \rightarrow \sigma\left(\varepsilon_{\perp}, t\right) .
$$

Точнее, имеется равномерная сходимость функции $\sigma\left(x, p_{x}, t\right)$ к $\sigma\left(\varepsilon_{\perp}, t\right)$ для любой последовательности $\Delta t$, если $\lim _{\delta \rightarrow 0} \delta^{2} \Delta t=0$.

\section{3. БОЛЬШИЕ ГЛУБИНЫ ПРОНИКНОВЕНИЯ: КОЛЕБАТЕЛЬНАЯ РЕЛАКСАЦИЯ С УЧЕТОМ ВКЛАДА АНГАРМОНИЗМА}

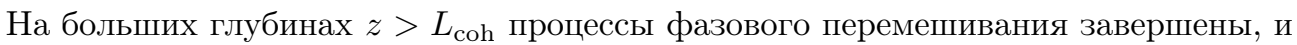
основную роль в эволюции системы играют диссипативные процессы, для описания которых удобно использовать неравновесную термодинамику квантовых систем.

Если подсистему КЧ моделировать гармоническими осцилляторами, то полный гамильтониан системы

$$
H_{1}=H_{\mathrm{ph}}+H_{\mathrm{p}}^{(\text {har })}+H_{\mathrm{int}}
$$

включает в себя $H_{\mathrm{ph}}=\sum_{q} \omega_{q} b_{q}^{+} b_{q}$ - гамильтониан решетки $\left(b_{q}^{+}, b_{q}\right.$ - операторы Бозе, $\omega_{q}$ - энергия кванта упругих колебаний $), H_{\mathrm{p}}^{(\text {har })}=\sum_{s} \omega_{s} a_{s}^{+} a_{s}$ - гамильтониан КЧ в гармоническом приближении и $H_{\mathrm{int}}$ - гамильтониан взаимодействия КЧ с тепловыми колебаниями решетки. Оператор $H_{\mathrm{int}}$ определяется в сопровождающей системе

5 Теоретическая и математическая физика, т. 167, № 1, 2011 г. 
координат, так что $\omega_{s}=\Omega s$ - энергия осциллятора, колеблющегося в $x$-направлении, $s-$ колебательное квантовое число, $a_{s}^{+}$и $a_{s}$ - операторы Ферми [8].

Будем рассматривать атомную плоскость низкоиндексного канала как совокупность атомных $x$-цепочек и выделим одну из них, а именно ту, которая является ближайшей в данный момент к частице. Тогда

$$
H_{\mathrm{int}}=\varkappa_{0} \sum_{\langle d\rangle} h\left(X_{\mathrm{c}}\right) u\left(X_{\mathrm{c}}+d\right)
$$

где $\varkappa_{0}$ - константа связи частицы с решеткой, $u$ - смещение атома решетки относительно узла, $h\left(X_{\mathrm{c}}\right)$ - смещение частицы относительно середины канала $X_{\mathrm{c}}, X_{\mathrm{c}}+d-$ координаты ближайших атомов стенки. Переходя в представление вторичного квантования, гамильтониан (9) запишем в виде

$$
H_{\mathrm{int}}=\sum_{s, q} \varepsilon_{q}(s, s+1) \exp \left\{-i\left(q-\frac{2 \pi}{d}\right) X_{\mathrm{c}}\right\} a_{s}^{+} a_{s}\left(b_{q}^{+}+b_{-q}\right)+\text { K.c. }
$$

Здесь

$$
\varepsilon_{q}(s, s+1)=I_{q}(s+1)^{1 / 2}, \quad I_{q}=-\frac{i \varkappa_{0}}{\sqrt{\tilde{N} M \omega_{q} m \Omega}},
$$

$M$ и $m$ - массы атома решетки и КЧ, $\tilde{N}$ - число атомов в $x$-цепочке.

Перейдем к изучению системы ангармонических осцилляторов. Для этого достаточно ввести потенциал $V(X)$ атом-атомного взаимодействия, включающий члены более высокого порядка, чем $h^{2}(X)$, в частности, потенциал

$$
V\left(X_{\mathrm{c}}\right)=\frac{1}{2} \widetilde{U}_{0}\left[1-\cos \frac{2 \pi}{d} h\left(X_{\mathrm{c}}\right)\right]
$$

Тогда, ограничиваясь членами порядка $h^{4}$, запишем полный гамильтониан в виде

$$
H_{2}=H_{\mathrm{ph}}+H_{\mathrm{p}}^{(\mathrm{har})}+H_{\mathrm{p}}^{(\mathrm{anh})}+H_{\mathrm{int}} .
$$

Здесь

$$
\begin{aligned}
& H_{\mathrm{p}}^{(\mathrm{anh})}=\lambda \sum_{\substack{l m f g \\
\eta \nu \rho \sigma}} \Phi_{l m f g}^{\eta \nu \rho \sigma} \exp \left\{i \frac{2 \pi}{d} X_{\mathrm{c}} \Lambda\right\} a_{l}^{+} a_{l+\eta} a_{m}^{+} a_{m+\nu} a_{f}^{+} a_{f+\rho} a_{g}^{+} a_{g+\sigma}, \\
& \Phi_{l m f g}^{\eta \nu \rho \sigma}=-\frac{2}{3}\left(\frac{\pi}{d}\right)^{4} \widetilde{U}_{0}\langle l|\delta x| l+\eta\rangle\langle m|\delta x| m+\nu\rangle\langle f|\delta x| f+\rho\rangle\langle g|\delta x| g+\sigma\rangle .
\end{aligned}
$$

В данных формулах дискретные переменные $\eta, \nu, \rho, \sigma$ принимают два значения +1 и $-1,\langle l|\delta x| l+\eta\rangle$ - матричный элемент смещения КЧ относительно центра канала, $\Lambda=\eta+\nu+\rho+\sigma$. Безразмерный формальный параметр $\lambda$ указывает порядок ангармонического вклада, и в окончательном выражении этот параметр будет положен равным единице.

Для описания системы в рамках квантовой теории колебательной релаксации необходимо использовать квазиравновесный статистический оператор $\rho_{\ell}(t)$. Если 
термодинамические параметры системы изменяются достаточно медленно, то можно ограничиться использованием стационарного статистического оператора $\rho_{\ell}$, которому в классическом случае соответствует совместная плотность вероятности. Введение $\rho_{\ell}$ позволяет дать определение двухвременной функции Грина [9]:

$$
G_{s s^{\prime}}(t)=\left\langle\left\langle a_{s}^{+}(t) a_{s^{\prime}}(t) ; B\right\rangle\right\rangle=\frac{1}{i} \theta(t)\left\langle\left[a_{s}^{+}(t) a_{s^{\prime}}(t), B\right]\right\rangle,
$$

где $B$ - термодинамическая сила, $\langle A\rangle=\mathrm{Sp}\left(\rho_{\ell} A\right),[A, B]$ - коммутатор.

Построим уравнение движения для функции (13) с учетом совместного воздействия на частицы неупругого рассеяния (10) и ангармонизма (12). Нетрудно показать, что в это уравнение входят функции Грина более высокого порядка, в частности функция $\left\langle\left\langle a_{s}^{+}(t) a_{s^{\prime}}(t), H_{2}(t)\right] ; B\right\rangle$, которую, раскрывая коммутатор, можно представить в виде суммы функций Грина вида $\left\langle\left\langle a_{s}^{+} a_{l+\eta} a_{m}^{+} a_{m+\nu} a_{f}^{+} a_{f+\rho} a_{g}^{+} a_{g+\sigma} ; B\right\rangle\right.$. Последняя является поправкой к равновесной функции

$$
\left\langle a_{s}^{+} a_{l+\eta} a_{m}^{+} a_{m+\nu} a_{f}^{+} a_{f+\rho} a_{g}^{+} a_{g+\sigma}\right\rangle,
$$

которая с учетом нормировки одночастичного распределения на единицу может быть аппроксимирована выражением

$$
\left\langle a_{s}^{+} a_{g+\sigma}\right\rangle \delta_{l+\eta, m} \delta_{m+\nu, f} \delta_{f+\rho, g} .
$$

Согласно работам [9]-[11] изменение распределения (14) под влиянием возмущения, обусловленного термодинамической силой $B$, выражается через функцию Грина, которая связывает оператор, вошедший в функцию (14), и силу $B$. Поэтому соответствующая поправка с учетом (15) имеет вид

$$
\left\langle\left\langle a_{s} a_{l+\eta} a_{m}^{+} a_{m+\nu} a_{f}^{+} a_{f+\rho} a_{g}^{+} a_{g+\sigma} ; B\right\rangle\right\rangle=\left\langle\left\langle a_{s}^{+} a_{g+\sigma} ; B\right\rangle\right\rangle \delta_{l+\eta, m} \delta_{m+\nu, f} \delta_{f+\rho, g} .
$$

Анализ показал, что если при выводе использовать выражение (16), то в уравнение для функции (13) войдут функции вида $\left\langle\left\langle a_{s}^{+} a_{g+\sigma} ; B\right\rangle\right.$, а именно функции Грина

$$
\begin{gathered}
\left\langle\left\langle a_{s}^{+}(t) a_{g+\sigma}(t) ; B\right\rangle\right\rangle, \quad\left\langle\left\langle a_{s}^{+}(t) a_{s^{\prime}+\Lambda}(t) ; B\right\rangle\right\rangle, \quad\left\langle\left\langle a_{s+\Lambda}^{+}(t) a_{s^{\prime}}(t) ; B\right\rangle\right\rangle, \\
\left\langle\left\langle a_{s-1}^{+}(t)\left\{b_{q}^{+}(t)+b_{-q}(t)\right\} a_{s^{\prime}}(t) ; B\right\rangle\right\rangle, \quad \ldots
\end{gathered}
$$

На основе уравнений движения для этих функций можно получить замкнутое уравнение для $G_{s s^{\prime}}(t)$, применяя следующие приближения: предполагается, что взаимодействие частицы с решеткой является слабым, тогда коэффициенты перед $G_{s s^{\prime}}(t)$ достаточно вычислить с точностью до членов порядка $\varkappa_{0}^{2}$; вклад ангармонизма вычисляется с точностью до $\lambda^{2}$.

После ряда преобразований, выполненных с учетом данных приближений, замкнутое уравнение для поправки $\delta G_{s s^{\prime}}(t)=G_{s s^{\prime}}(t)-G_{s s^{\prime}}^{(0)}(t)$ к функции Грина нулевого порядка принимает достаточно простой вид. Запишем его для фурье-компоненты поправки $\delta G_{s s^{\prime}}(E)$. Имеем

$$
\begin{aligned}
(E+ & \left.\omega_{s s^{\prime}}\right) \delta G_{s s^{\prime}}(E)=-2 \pi i \lambda^{2} 2 \tilde{N}(0) \sum_{q}\left\{\left[\left|\varepsilon_{q}(s, s+1)\right|^{2} \times\right.\right. \\
& \left.\left.\times \tilde{J}\left(s, s+1 ; s^{\prime} s^{\prime}+1 \mid E\right)\right]-\left[s \rightarrow s-1 ; s^{\prime} \rightarrow s^{\prime}-1\right]\right\} \delta\left(\omega_{q}-\Omega\right) .
\end{aligned}
$$


Здесь

$$
\begin{gathered}
2 \tilde{J}\left(s, s+1 ; s^{\prime}, s^{\prime}+1 \mid E\right)=f(\xi) \sum_{\eta \nu \rho \sigma} \Lambda^{-2} w_{s}(\eta, \nu, \rho, \sigma)- \\
-[f(\xi)+1] \sum_{\eta \nu \rho \sigma} \Lambda^{-2} w_{s+1}(\eta, \nu, \rho, \sigma), \\
\Omega^{2} w_{s}(\eta, \nu, \rho, \sigma)=2 \sum_{\eta^{\prime} \nu^{\prime} \rho^{\prime} \sigma^{\prime}} \Phi_{s, s+\eta, s+\eta+\nu, s+\eta+\nu+\rho, s+\Lambda}^{\eta \nu \rho \sigma} \times \\
\times \Phi_{s, s+\eta^{\prime}, s+\eta^{\prime}+\nu^{\prime}, s+\eta^{\prime}+\nu^{\prime}+\rho^{\prime}, s+\Lambda^{\prime}}^{\eta^{\prime} \sigma_{\Lambda}^{\prime}},
\end{gathered}
$$

$f(\xi)=\left(e^{\xi}-1\right)^{-1}, \xi=\beta_{1} \Omega, \omega_{s s^{\prime}}=\omega_{s}-\omega_{s^{\prime}}, \tilde{N}(0)-$ плотность состояний КЧ.

При наличии конечной высоты барьера $\widetilde{U}_{0}=\Omega s_{0}$ каждый уровень $\omega_{s}$ (на диаграмме, на которой изображаются одновременно энергетический спектр и потенциальная яма $\left.U_{\mathrm{pl}}(x)\right)$ размывается в зону шириной $\Delta \omega_{s}$. Поэтому каждое состояние с энергией $\omega_{s}$ следует дополнить набором состояний осциллятора с энергиями в интервале $\Delta \omega_{s}<\Omega$. Если использовать зонную структуру параболического потенциала конечной высоты $\widetilde{U}_{0}$, то надбарьерные состояния $s>s_{0}$ можно также рассматривать как осцилляторные, причем ширина надбарьерных зон больше ширины подбарьерных.

Учитывая принятую нами зонную структуру, необходимо заменить функцию (13) на функцию Грина $G_{s \bar{s}}(t)=\left\langle\left\langle a_{s}^{+}(t) a_{\bar{s}}(t) ; B\right\rangle\right\rangle$, соответствующую неравновесной добавке к функции распределения частиц в $s$-й зоне (здесь $a_{\bar{s}}=a\left(\omega_{s}+\Delta \omega_{s}\right)$ ).

Согласно квантовой теории переходов в твердых телах [12] полная вероятность деканалирования - выхода частицы из подбарьерного состояния ямы - пропорциональна вероятности последнего "шага", т. е. вероятности перехода $s_{0} \rightarrow s_{0}+1$. Деканалированные частицы могут быть снова захвачены в канал (реканалирование), при этом вероятность захвата пропорциональна вероятности обратного перехода $s_{0}+1 \rightarrow s_{0}$. Чтобы максимально упростить картину деканалирования или реканалирования, можно использовать двухуровневую систему, включающую уровень потенциального барьера $s_{0}$ и уровень нижнего надбарьерного состояния $s_{0}+1$. В этой модели, используя уравнения для функции Грина (18), построим замкнутое уравнение для поправки к функции Грина нулевого (по взаимодействию) порядка.

Если перейти к фурье-компонентам функций Грина, то окончательный вид уравнения таков:

$$
E\left\{\delta G_{s_{0} \bar{s}_{0}}(E)-\delta G_{s_{0}+1, \bar{s}_{0}+1}(E)\right\}=i \tilde{N}(0) \frac{1}{\tau_{\varepsilon_{\perp}}\left(\beta_{1}\right)}\left(\frac{1}{\Omega \tau_{s_{0}}}-e^{\xi} \frac{1}{\Omega T_{s_{0}+1}}\right) .
$$

В это уравнение, описывающее колебательную релаксацию ангармонических осцилляторов в двухуровневой модели, вошли следующие физические величины:

$$
\frac{1}{\tau_{\varepsilon_{\perp}}\left(\beta_{1}\right)}=2 \pi \sum_{q}\left|\varepsilon_{q}\left(s_{0}, s_{0}+1\right)\right|^{2} f\left(\beta_{1}\right) \delta\left(\omega_{q}-\Omega\right)
$$

- время релаксации поперечной энергии КЧ в условиях однофононных процессов,

$$
\begin{aligned}
\frac{1}{\Omega \tau_{s}} & =\sum_{\eta \nu \rho \sigma}\left(\frac{1}{\Lambda}\right)^{2} w_{s}^{\eta \nu \rho \sigma} \delta\left(s-s_{0}-\Lambda+1\right), \\
\frac{1}{\Omega T_{s}} & =\sum_{\eta \nu \rho \sigma}\left(\frac{1}{\Lambda}\right)^{2} w_{s}^{\eta \nu \rho \sigma} \delta\left(s-s_{0}-\Lambda\right)
\end{aligned}
$$


- перенормировочные множители, обусловленные ангармоническим взаимодействием, в условиях деканалирования и реканалирования частиц. С учетом перенормировки полные частоты релаксации ангармонических осцилляторов равны

$$
\begin{array}{ll}
\frac{1}{\tau_{\varepsilon_{\perp}}} \cdot \frac{1}{\Omega \tau_{s_{0}}} & \text { (прямой переход } s_{0} \rightarrow s_{0}+1, \text { деканалирование) }, \\
\frac{1}{\tau_{\varepsilon_{\perp}}} \cdot \frac{1}{\Omega T_{s_{0}+1}} & \text { (обратный переход } s_{0}+1 \rightarrow s_{0} \text { реканалирование) }
\end{array}
$$

Коэффициент деканалирования определяет скорость выхода частиц из режима направленного движения в результате неупругого рассеяния [2]. Методами неравновесной статистической термодинамики для частиц, которые моделируются как гармонические осцилляторы, скорость выхода за счет их взаимодействия с тепловыми колебаниями решетки проанализирована в работе [8]. С учетом вклада ангармонизма колебаний частиц между стенками канала (23) скорость выхода может быть записана в виде

$$
R^{(\mathrm{anh})}=\mathrm{const} \cdot \frac{1}{\tau_{\varepsilon_{\perp}}\left(\beta_{1}\right)} K\left(F_{21}\right) \frac{1}{\Omega \tau_{s_{0}}} \zeta^{4} .
$$

В данной формуле $K\left(F_{21}\right) \sim\left(e^{F_{21} \widetilde{U}_{0}}+1\right)^{-1}-$ статистический множитель, зависящий от $1 / F_{21}-$ поперечной квазитемпературы КЧ [13];

$$
\frac{1}{\tau_{\varepsilon_{\perp}}\left(\beta_{1}\right)} \simeq \frac{\varkappa_{0}^{2} l^{2}}{(2 \pi)^{2} g_{\mathrm{c}}} \varphi\left(\beta_{1}\right) \widetilde{\Theta}\left(v_{0}\right)
$$

- частота колебательной релаксации; $\widetilde{\Theta}\left(v_{0}\right) \simeq 1 / v_{0} a_{\text {тF }} m$ - минимальный угол рассеяния в борновском приближении; $\varphi\left(\beta_{1}\right)=\left(1-e^{-\xi}\right)^{-1}-$ статистический множитель, зависящий от температуры решетки $1 / \beta_{1}, \zeta=\left(\Omega / \varepsilon_{\perp}^{*}\right), \varepsilon_{\perp}^{*}=\widetilde{U}_{0}+\bar{\varepsilon}-$ пороговая энергия, на которой "обрезано" энергетическое распределение частиц в квазиклассическом приближении [8], $\bar{\varepsilon} \ll \Omega, g_{\text {c }}$ - силовая постоянная. Отметим, что вычисление кинетического коэффициента (24) выполнено в сопровождающей системе координат, в которой фигурируют только переменные поперечного движения частиц. Единственный множитель, отражающий факт продольного движения, - это минимальный угол рассеяния; действительно, $\widetilde{\Theta} \sim 1 / v_{0}$.

\section{4. ЗАКЛЮЧЕНИЕ}

В классической теории каналирования подсистема КЧ описывается с помощью совместной плотности вероятности. На малых глубинах проникновения частиц в кристалл неупругое рассеяние еще не эффективно, и негармоничность потенциала плоскостного канала приводит к наиболее заметным физическим последствиям. Действительно, градиент негармонического потенциала в уравнениях (1) и (6) определяет сглаживание пространственной плотности распределения частиц. Естественно, никакие представления об установлении квазиравновесия невозможны, и все расчеты выполняются методом численного интегрирования (см. рис. 1-3). 
Квазиравновесное, квазистационарное распределение КЧ устанавливается после затухания осцилляций плотности за счет обмена колебательной энергией в подсистеме частиц. Перераспределение энергии и установление квазиравновесия КЧ обусловлено ангармоническими эффектами четвертого порядка, при этом ангармонические поправки к гармоническому гамильтониану рассматриваются нами как возмущение, которое вызывает переходы частиц из одного состояния в другое. Такие переходы принято связывать с так называемыми “столкновениями", чтобы подчеркнуть аналогию с другими явлениями переноса [6].

В общем случае достижение квазиравновесия КЧ означает одновременное формирование как в $x$-пространстве, так и в $p_{x}$-пространстве квазиравновесного распределения. Однако в большинстве случаев наступление равновесия в импульсном пространстве может и не представлять особого интереса. Например, при расчете потока частиц по поперечному сечению канала нас прежде всего интересует общее число частиц, пересекающих заданный элемент площади, независимо от того, каково направление скорости этих частиц. Тем не менее даже в этом случае, как показывает анализ [14], асимметрия в угловом распределении слабо сказывается на равномерном заполнении доступной области канала.

Дальнейшая эволюция подсистемы КЧ после установления квазиравновесия описывается на основе кинетического уравнения (18), которое включает в себя совместное воздействие неупругого рассеяния на тепловых колебаниях решетки и ангармонизма колебаний частиц в канале. Поскольку неупругое рассеяние и обмен колебательной энергией, обусловленный взаимодействием (12), перекрываются, в соотношении (18) динамические эффекты столкновений представлены в форме интерференции указанных процессов. Другими словами, более слабое воздействие, ангармонизм, дает всего лишь перенормировку основной частоты колебательной релаксации. И только в том случае, когда вклад ангармонизма можно считать заметным, $\Omega \tau_{s_{0}}<1$, перенормировочный множитель увеличивает скорость релаксации колебательной энергии. Рассмотренный нами эффект аналогичен перекрыванию спин-спинового и спин-решеточного взаимодействий в теории магнетизма твердых тел [15], а также интерференции воздействий в теории колебательной релаксации в газах [5].

\section{Список литературы}

[1] Й. Линдхард, УФН, 99:2 (1969), 249-296.

[2] Е.-Х. Оцуки, Взаимодействие зарлженных частии, с твердыми телами, Мир, М., 1985.

[3] Т. В. Оуглтри, Microsoft Windows XP: Теория и практика эффективной работь в операционной системе, ДиаСофт, СПб., 2002.

[4] Ю. Каган, Ю. В. Кононец, ЖЭТФ, 66:5 (1974), 1693-1711.

[5] Б. Ф. Гордиец, А.И. Осипов, Е. В.Ступоченко, Л.А. Шелепин, УФН, 108:12 (1972), 655-699.

[6] Н. Ашкрофт, Н. Мермин, Физика твердого тела, т. 2, Мир, М., 1979.

[7] Н. Н. Боголюбов, Избранные труды, т. 2, Наукова думка, Киев, 1970.

[8] Ю. А. Кашлев, ТМФ, 19:3 (1974), 400-413.

[9] Д.Н. Зубарев, “Современные методы статистической теории неравновесных процессов", Итоги науки и техн. Сер. Соврем. пробл. мат., 15, ред. Р. В. Гамкрелидзе, ВИНИТИ, М., 1980, 131-226. 
[10] M. Gomes-Rodriquez, Phys. Rev. B, 2:10 (1970), 4262-4272.

[11] Ю. А. Кашлев, Неравновесная статистическая теория скоростей диффузионных процессов в твердых телах (диффузионного деканалирования и диффузионной миграчии), Автореф. дис. ... докт. физ.-мат. наук, Матем. ин-т им. В. А. Стеклова, М., 1982.

[12] J. A. Sussmann, J. Phys. Chem. Solids, 28:9 (1967), 1643-1648.

[13] Ю. А. Кашлев, ТМФ, 142:1 (2005), 112-126.

[14] Н. П. Калашников, В. С. Ремизович, М. И. Рязанов, Столкновения быстрых заряженных частии в твердых телах, Атомиздат, М., 1980.

[15] М. Гольдман, Спиновая температура и ЯМР в твердых телах, Мир, М., 1972.

Поступила в редакцию 28.06.2010, после доработки 27.10.2010 